\title{
Corela
}

Cognition, représentation, langage

5-2 | 2007

Vol. $5, \mathrm{n}^{\circ} 2$

\section{Une propriété pragmatique du past perfect}

\section{Emmanuelle Roussel}

\section{OpenEdition}

\section{Journals}

Édition électronique

URL : http://journals.openedition.org/corela/397

DOI : $10.4000 /$ corela.397

ISSN : 1638-573X

\section{Éditeur}

Cercle linguistique du Centre et de l'Ouest - CerLICO

\section{Référence électronique}

Emmanuelle Roussel, « Une propriété pragmatique du past perfect », Corela [En ligne], 5-2 | 2007, mis en ligne le 30 décembre 2007, consulté le 19 avril 2019. URL : http://journals.openedition.org/ corela/397 ; DOI : 10.4000/corela.397

Ce document a été généré automatiquement le 19 avril 2019

\section{(c) (i) (2)(2)}

Corela - cognition, représentation, langage est mis à disposition selon les termes de la licence Creative Commons Attribution - Pas d'Utilisation Commerciale - Partage dans les Mêmes Conditions 4.0 International. 


\title{
Une propriété pragmatique du past perfect $^{1}$
}

\author{
Emmanuelle Roussel
}

\section{Introduction}

1 Le point de vue adopté ici est que le past perfect peut se prêter à une analyse pragmatique, parallèlement à ses propriétés syntaxiques et sémantiques.

2 La présentation traditionnelle du past perfect ne rend pas toujours compte de la diversité des occurrences, et l'étude de certains énoncés choisis indique que le past perfect a des caractéristiques qui lui sont propres. Had-en relève alors d'unprocessus de codification dans l'énoncé des motivations de l'acte de communication : l'énonciateur recourt au past perfect afin d'effectuer une focalisation sur la notion prédicative quand il s'agit de souligner qu'elle est contraire aux attentes du co-énonciateur, voire à ses propres attentes parfois.

3 D'un point de vue cognitif, l'argument est parfaitement recevable, si l'on s'appuie par exemple sur l'une des définitions de l'intelligence (ou des intelligences selon les courants); on y trouve l'intelligence interpersonnelle "permettant les interactions sociales et la compréhension des motivations, émotions et comportements d'autrui » (H. Gardner 1983 in Grubar 2002).

\section{Quelques caractéristiques du past perfect}

4 Traditionnellement, le past perfect est l'expression d'un accompli (-en) réactualisé dans le discours par sa juxtaposition à un autre élément actualisateur : l'auxiliaire. Il est présenté dans les grammaires comme la translation du present perfect ou du prétérit dans la sphère temporelle du passé. Dans la Théorie des Opérations Enonciatives, had, opérateur de localisation, marque une différenciation entre le moment de l'événement et celui de 
l'énonciation, et -en fournit l'extérieur de la notion du procès considéré. D'où les points de vue aspectuels d'état résultant, de bilan :

(1) The Pickwick Papers came out in monthly parts. Of the first number only four hundred were sold, but by the time the fifteenth came out, the number had risen to forty thousand. (C. Dickens par J. Bouscaren 1982 : 101) ou d'explication :

(2) My mother fell on the ice outside our supermarket and broke her hip. They hadn't even bothered to put salt down. Can we sue for damages? (Hartlepool par J. Bouscaren 1982 : 87) regroupés sous le terme de « commentaire » (J. Bouscaren 1982) qui s'ajoutent à la valeur temporelle d'antériorité de la forme².

Il est possible de proposer une autre approche de had-en qui étudie la véritable nature des relations entre le procès et les repères en présence, mettant ainsi en évidence les situations objectives que la forme permet de construire (situations qui recoupent inévitablement les valeurs de bilan, d'aspect résultant, et d'antériorité, mais le but recherché étant de repenser l'analyse du past perfect, ces valeurs ne sont plus considérées comme telles). Had-en place en effet plusieurs repères ; prioritairement $T_{1}$ (repère interne au récit), mais également $\mathrm{T}_{2}$ (passé par rapport à $\mathrm{T}_{1}$ ), $\mathrm{T}_{3}$ (passé dans le passé par rapport à $T_{1}$ ), voire $T_{4}, T_{5}, T_{6}$, selon le même principe, ou encore $T_{0}$. Partant, référer à la forme en seuls termes de translation d'un present perfect ou d'un prétérit dans la sphère temporelle du passé n'en fournit pas une présentation satisfaisante. Cette approche objective révèle un emploi non marqué de had-en, la continuité (d'un procès entre deux points du passé ; 43 \% d'un corpus de 15508 occurrences) et un emploi marqué, la rupture (39 \% du même corpus), s'appuyant sur de nombreux facteurs spécifiques (aspect interne du lexème verbal, nature et référence des constituants, des personnages, présence ou non d'adverbiaux, de quantifieurs, de déterminants, de compléments d'objet - pluralisés ou non, de propositions subordonnées, etc. ${ }^{3}$ ). Le past perfect peut, dans un même énoncé, illustrer la continuité, la rupture, ou bien la succession des deux valeurs $(C+R=2 \%)$. Les relations observées ${ }^{4} s^{\prime}$ organisent sur un arrière-plan énonciatif invariablement continu ${ }^{5}$ qui les rapporte à $\mathrm{T}_{1}$ (en conditions d'emploi de had-en dans un cadre temporel passé) et forment donc un système basé sur deux schémas notionnels : continuité versus rupture, correspondant à 5 principaux cas de figure ${ }^{6}$ :

- $\mathrm{T}_{2} \rightarrow \mathrm{T}_{1}$ (« continuité entre $\mathrm{T}_{2}$ et $\mathrm{T}_{1}$ »;1870 occurrences $=41,73 \%$ - sur un total de 4481 - $c f$. note 4) :

(3) After tea Tony wrote to Brenda; he had taken to writing letters in the past few weeks. (E. Waugh 1988 : 99)

- $\mathrm{T}_{3} \rightarrow \mathrm{T}_{2} \neq \mathrm{T}_{1}$ (« continuité entre $\mathrm{T}_{3}$ et $\mathrm{T}_{2}$ et rupture entre $\mathrm{T}_{2}$ et $\mathrm{T}_{1}$ » $; 1081=24 \%$ ):

(4) 'I would not', she said sadly. 'But I had been brought up to that life; and it was second nature to me.' (T. Hardy $1972: 68)$ La femme qui parle est adulte en $T_{1}$.

- $\mathrm{T}_{2} \neq \mathrm{T}_{1}$ (« rupture entre $\mathrm{T}_{2}$ et $\mathrm{T}_{1} » ; 924=20,62 \%$ ):

(5) I hinted I had had an affair with her thirteen years ago but this was not mentioned in print. (V. Nabokov $1980: 75$ )

- $\mathrm{T}_{3} \neq \mathrm{T}_{2} \neq \mathrm{T}_{1}$ ( « rupture entre $\mathrm{T}_{3}$ et $\mathrm{T}_{2}$ et entre $\mathrm{T}_{2}$ et $\mathrm{T}_{1} » ; 284=6 \%$ ):

(6) He had pushed his straw hat down on his head before he hooked the fish and it was cutting his forehead. (E. Hemingway 1989 : 37)

$8 \mathrm{~T}_{3}=$ He had pushed his straw hat down on his head; $\mathrm{T}_{2}=$ before he hooked the fish; $\mathrm{T}_{1}=$ it was cutting his forehead

- $\mathrm{T}_{3} \rightarrow \mathrm{T}_{2} \rightarrow \mathrm{T}_{1}$ (« continuité entre $\mathrm{T}_{3}$ et $\mathrm{T}_{2}$ et entre $\mathrm{T}_{2}$ et $\mathrm{T}_{1}$ » $73=1,63 \%$ ): 
(7) She had indeed figuredin his child mind, and even still, more dimly as an angel.

(I. Murdoch 1986 : 54)

\subsection{Continuité et Rupture}

Dans cette perspective, la continuité souligne la validité du procès en tel ou tel repère. C'est là le sémantisme le plus fréquemment rencontré, ce qui nul doute justifie la vision du past perfect comme une translation du present perfect dans le passé. Cette continuité est de deux natures : référentielle ou énonciative. Le procès se poursuit jusqu'au repère ou bien l'énonciateur reconnaît et s'approprie mentalement la notion attachée au prédicat (la validation de la relation prédicative « a laissé des traces »). La rupture indique quant à elle que le procès est révolu en tel ou tel repère et illustre deux valeurs: elle est référentielle " pure » exprimant une rupture par rapport à un « $T$ » quelconque, ou elle est référentielle tout en s'accompagnant d'une continuité énonciative jusqu'en ce même « $\mathrm{T} »$.

Cette analyse indique que la continuité énonciative est commune aux deux valeurs. Elle peut s'expliquer par l'opération de localisation dont témoigne have en repérant le procès dans la sphère du sujet de la relation prédicative. Ce faisant, on s'appuie sur les constituants en leur appliquant des propriétés sémantiques. La continuité énonciative peut également être due à l'origine nominale du participe passé qui en fait une entité stable. Elle peut enfin être intrinsèquement liée à l'organisation des activations cérébrales présidant à l'énonciation (cf. note 5). C'est une question qui ne sera pas développée ici.

13 Il s'ensuit que la rupture devient, dans ce système, la valeur signifiante puisque marquée (le commentaire relevé dans les années 1980 procéderait de cette rupture). Par ailleurs, ces deux valeurs se retrouvent dans des contextes présents, où had-en est repéré par rapport à $\mathrm{T}_{0}$. L'étude de ces énoncés révèle un fonctionnement intéressant de la forme.

\subsection{Had-en en $\mathrm{T}_{0}(12=0,26 \%)$}

Voici quelques énoncés typiques en contexte présent :

(8) 'Aren't you going to give Mr Scott one day's rest?'

'Wish I could, Lizzie. 'Fraid I can't. As long as he can stand up we must use him.'

'Well, I've had one Europe evening, at least... By Jove, I'd nearly forgotten! What do I do about those babies of mine?'? (R. Kipling $1972: 90$ )

(9) 'I'll tell you something very odd,' he said. 'Who do you think is here?

'Who?'

'Old friend Beaver.'

'Why's that odd, particularly?'

'Oh, I don't know. I'd forgotten all about him, hadn't you? D'you think he sent a telegram as he did to us?' (E. Waugh : 62)

(10) 'There are things I know, though I don't know how I know them, and things I remember. Certain things are established in my mind as facts, but no picture attaches to them; on the other hand there are pictures unverified by any fact which recur obsessively, like the landscape of a dream.

The facts I owe to my diary (...). The last few entries are in code. Not a pretence 
code (...) but a real one like Pepy's - perhaps I had heard of his. (L.P. Hartley 1958 :

32)

(11) I'm afraid he must have noticed the alteration in my manner: the sidesman and the verger had shown much more pride of bearing than I did.'

'Does Marian call you Leo?' (L.P. Hartley : 72)

(12) They give me slow, uninterested looks.

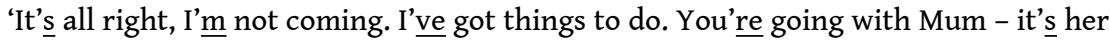
idea - on the bus.' And almost immediately their eyes, which only a second before had been full of reluctance, begin to show enthusiasm - and relief. (G. Swift 1982 : 56)

(13) He said, 'You've made mistakes. That beating up - Miss Cullen will never support you that I had stolen the car. And then the shooting - I didn't fing the bullet. Mis Cullen did. I am going to bring a charge....' (G. Greene 1971 : 93)

Ces énoncés illustrent toujours un double repérage : l'un en regard de $\mathrm{T}_{1}$ (-ed sur have), l'autre en regard de $\mathrm{T}_{0}$ (contexte d'énonciation). Le repérage par rapport à $\mathrm{T}_{0}$ fait apparaitre une forte influence de ce que l'on peut désigner par la pragmatique contextuelle, qui met en scène la connaissance que le lecteur a du roman, de l'intrigue, de la nature des relations entre les personnages, des évolutions de ces relations, etc. La simple mention d'un fait amène ce lecteur à agencer inconsciemment des repères implicites. Cette situation s'observe aussi bien pour la continuité que pour la rupture, les deux valeurs décrites plus haut. Ce ne sont donc pas non plus des facteurs temporels de l'ordre de l'antériorité par rapport à $\mathrm{T}_{1}$ qui peuvent en priorité déclencher le past perfect. Son occurrence en $\mathrm{T}_{0}$ est révélatrice à cet égard.

\subsection{Le facteur « pragmatique contextuelle»}

L'influence du facteur pragmatique est indiscutable. C'est ainsi que de manière empirique, sur $284 \quad(6 \%)$ énoncés illustrant le repérage $T_{3} \neq T_{2} \neq T_{1}$, comme (14):

(14) Herman was an only child. His father Reb Shmuel Leib Broder, a follower of the Rabbi of Hushatin, was a wealthy man who owned several houses in Tzivkev. He hired a rabbi to instruct his son in Jewishness and a Polish tutor to teach him secular subjects. Reb Shmuel Leib hoped his only son would become a modern rabbi. Herman's mother who had attended a German gymnasium in Lemberg, wanted her son to become a doctor. At nineteen, Herman went to Warsaw, passed his matriculation exams, and enrolled in the school of philosophy at the university [...]. (I.B. Singer $1989: 29$ )

$\mathrm{T}_{3}=$ in Lemberg $; \mathrm{T}_{2}=$ wanted $; \mathrm{T}_{1}=$ Herman adulte

$221(78 \%)$ reposent sur la pragmatique interne au roman (au sens de « connaissances partagées par le narrateur et le lecteur »).

Le phénomène intervient également pour (15), de repérage $T_{3} \rightarrow T_{2} \neq T_{1}$ :

(15) Anger, violence, death, seemed natural to this vast, harsh country [...], wondering why everyone was so late, to find Mary Turner lying murdered on the verandah, and the police boys outside, guarding the houseboy; and Dick Turner muttering and stumbling through the puddles, mad, but apparently harmless. Things he had not understood he understood now, and he was ready to talk about them.(D. Lessing $1989: 19)$

Le verbe se charge d'y véhiculer la continuité (aspect lexical), tandis que la rupture y est bien signifiée au moyen de la pragmatique contextuelle qui s'appuie sur le hiatus cognitif entre now $\left(\mathrm{T}_{1}\right)$ qui nous est donné, et un moment autre, ie not now, donc then. Le renvoi à un lieu, un personnage, un fait ou des circonstances particulières suffit dans la totalité 
des énoncés à déclencher la rupture. En effet, hormis la présence d'un adverbe, d'une proposition relative, d'une datation plus ou moins précise renvoyant au révolu ou d'une forte détermination, le past perfect de rupture fonctionne à la seule mention de sujets, d'objets (grammaticaux) ou de déterminants possessifs dont les référents s'inscrivent dans le révolu par rapport au moment de l'énonciation :

(16) The effect upon him of his mother, 'the girl from far away', was something more complex. [...] Her name was Teresa Maxton O'Neill, a Catholic, born in Dunedin of Irish immigrant parents. She had seen the great ocean seals basking on golden seaweed at the end of the world. (I.Murdoch : 54)

(17) Herman had hidden himself first in Tzivkev, then at Yadwiga's in Lipsk, [...]. Weeks had passed without his seeing the light of day. His eyes had grown accustomed to darkness; his feet had become numb with disuse. He had been eaten by insects, field mice, rats. He had developed a high fever and Yadwiga had cured him with herbs she picked in the fields and vodka she stole from her mother. (I.B. Singer : 28)

(18) They might take their ideas of the other world - of professional men and working people - partly from melodrama. It was wrong to underestimate the ignorance of the ruling class. Marie-Antoinette had said of the poor, 'Can't they eat cake?'(G. Greene : 53)

La rupture quant à elle est implicite ou explicite. Elle agit de deux manières : sa présence est immédiatement «perçue », voire « détectée », dans le même temps qu'elle procure un cadre à l'énoncé dans lequel vient se positionner la continuité :

(19) Gertrude Coppard had watched him, fascinated. (D.H. Lawrence 1948 : 17)

- continuité implicite : [watch] - verbe de processus (procès duratif)

- rupture implicite : Coppard désigne l'héroöne sous son nom de jeune fille. En $\mathrm{T}_{1}$, elle est Mrs Morel,

l'épouse du personnage auquel se rapporte him dans l'énoncé.

(20) She had been out of temper while dressing him. (E. Waugh : 100)

- continuité implicite : [be] - verbe d'état.

- rupture explicite : while dressing him.

(21) In Lipsk, she had done the work of a man. She had sown, mowed, threshed, planted potatoes, even sawed and chopped wood. (I.B. Singer: 10)

- continuité à la fois explicite et implicite : succession et réitération des différents procès.

- rupture explicite : in Lipsk.

L'étude du past perfect à partir de ce système continuité/rupture, et notamment l'analyse sémasiologique des énoncés, indique en outre l'effet ambivalent du contexte : il est indispensable à l'interprétation du past perfect dont il sélectionne cependant une valeur. Sans le contexte, le past perfect n'a aucune valeur précise (ou bien il en a plusieurs), avec le contexte il ne signifie plus qu'une chose. Partant, un énoncé n'est pas, en soi, restreint à un unique repérage et, chose remarquable, la continuité peut devenir rupture (et inversement), le référentiel peut devenir énonciatif (et inversement), sans modification 
aucune de la construction de l'énoncé. Les exemples suivants opposent la valeur de l'énoncé en contexte et celle qu'il prendrait hors contexte :

(22) The children had been taken from her. (I.B. Singer : 64)

Cet énoncé, initialement $T_{2} \neq T_{1}$ en contexte (It must have happened before 1941), devient $T_{2}$ $\rightarrow \mathrm{T}_{1}$ hors contexte.

(23) Walter Morel remained as he had stood, leaning on the table with one hand, looking blank. When he was sufficiently sure of his balance, he went across to her, swayed, caught hold o the back of her rocking-chair, almost tipping her out; then, leaning forward over her, and swaying as he spoke, he said, in a tone of wondering concern:

'Did it catch thee?'

He swayed again, as if he would pitch on to the child. With the catastrophe he had lost all balance. (D.H. Lawrence : 53)

$\mathrm{T}_{2} \rightarrow \mathrm{T}_{1}$ en contexte devient $\mathrm{T}_{2} \neq \mathrm{T}_{1}$ hors contexte.

(24) They were shocked, for the first week or so, by the way the natives were treated. They were revolted a hundred times a day by the casual way they were spoken of, as if they were so many cattle; or by a blow, or a look. They had been prepared to treat them as human beings. But they could not stand out against the society they were joining. It did not take them long to change. It was hard, of course, becoming as bad oneself. But it was not very long that they thought of it as 'bad'. And anyway, what had one's ideas amounted to? (D. Lessing: 18)

$\mathrm{T}_{3} \rightarrow \mathrm{T}_{2} \neq \mathrm{T}_{1}$ devient $\mathrm{T}_{2} \rightarrow \mathrm{T}_{1}$.

(25) And it was nine in the morning and I was eight years old, and all round me it was early summer... Lord knows how long we sat there. The morning stretched out and out, and the sun got higher and higher, and nobody had a bite... But the next moment there wasn't any doubt about it. The float dived straight down, I could still see it under the water, kind of dim red, and I felt the rod tighten in my hand..., The same moment all of us gave a yell of agony. The fish had slipped off the hook and fallen into the wild peppermint under the bank. (G. Orwell $1989: 63$ )

$\mathrm{T}_{3} \rightarrow \mathrm{T}_{2} \neq \mathrm{T}_{1}$ devient $\mathrm{T}_{2} \rightarrow \mathrm{T}_{1}$ et $\mathrm{T}_{2} \neq \mathrm{T}_{1}$.

(26) The past is a foreign country: they do things differently there. When I came upon the diary it was lying at the bottom of a rather battered red cardboard collarbox, in which as a small boy I kept my Eton collars. Someone, probably my mother, had filled it with treasures dating from those days...Something came and went between us: the intimate pleasure of recognition, the almost mystical thrill of early ownership - feelings of which, at sixty-odd, I felt ashamed... That night, in my bed, my smarting eyes shed tears for the first time. It was my second term at school; I had never been unpopular before, still less had I been systematically bullied, and I didn't know what to make of it... A late comer to school, I had uncritically accepted all its standards. (L.P. Hartley: 15)

$\mathrm{T}_{3} \neq \mathrm{T}_{2} \neq \mathrm{T}_{1}$ devient $\mathrm{T}_{2} \rightarrow \mathrm{T}_{1}$.

Les exemples sont innombrables. Le sémantisme final du past perfect est par conséquent indissociable des éléments contextuels. Par ailleurs, il est des cas où le past perfect est tout à fait capable de présenter un procès comme "coupé » référentiellement de $T_{1}$, et uniquement cela. Seule la rupture est alors représentée, d'où la possibilité qu'elle soit le trait atypique, discriminant, de la forme, trait qu'il faudrait transmettre (plutôt que celui de continuité qui pourtant lui semble si fortement attaché). C'est alors qu'intervient la prise en compte de ces processus « non spécifiquement linguistiques de l'interprétation des énoncés » (M. Bracops 2006 : 193), autrement dit des facteurs pragmatiques. En effet, il s'avère que ces valeurs de continuité et de rupture participent de ces processus non 
spécifiquement linguistiques et permettent au past perfect d'avoir une portée pragmatique.

\section{Analyse pragmatique}

L'existence de facteurs autres que grammaticaux transparaît déjà chez $\mathrm{J}$. Bouscaren (1982: 77) :

«On peut remarquer qu'il semble exister une relation beaucoup plus étroite entre

les deux procès quand had-en doit être maintenu. »

Ce qui mène à la valeur de « commentaire » avec laquelle est défini had-en.

Les valeurs de continuité et de rupture permettent pour leur part de préciser ce en quoi peut tenir le commentaire en question. Elles partagent en effet la propriété d'effectuer une focalisation sur la notion verbale, focalisation que l'énonciateur utilise dans différents buts. En ce sens, l'énoncé est un véritable acte illocutoire dont une lecture codique est incapable de rendre compte.

\subsection{La continuité pragmatique(23\% des énoncés illustrant la continuité sur un total de 69 énoncés)}

Quand bien même la continuité paraît indissociable du past perfect, elle n'est toutefois pas tangible pour autant. Contre toute attente, l'étude des facteurs déclencheurs de la continuité 9 peut être problématique, tant cette valeur semble "aller de soi» ${ }^{10}$. La continuité n'est pas toujours liée à un facteur grammatical explicite, comme le montrent les manipulations suivantes. Il est utile de rappeler ici que puisque le but est de mettre en évidence ce qui déclenche véritablement ces valeurs de continuité (ou plus loin de rupture), les énoncés sont cette fois étudiés de manière isolée, afin de comprendre précisément la manière dont les mécanismes à l'œuvre se mettent en place. Cela permet de rechercher à la fois, et ce au plus près de la forme, l'origine de l'interprétation et ce qui est susceptible de la modifier. C'est ainsi que nous allons découvrir une interaction inattendue entre la forme composée et les autres constituants des énoncés étudiés.

En (27) par exemple, la continuité n'est pas « déclenchée », elle est uniquement suggérée :

(27) He had sinned against Judaism, American law, morality. (I.B.Singer : 23)

(27a)People had sinned against Judaism, American law, morality.

$37 \rightarrow$ La continuité en $\mathrm{T}_{1}\left(\mathrm{~T}_{2} \rightarrow \mathrm{T}_{1}\right)$ et la rupture avec $\mathrm{T}_{1}\left(\mathrm{~T}_{3} \rightarrow \mathrm{T}_{2} \neq \mathrm{T}_{1}\right)$ peuvent toutes deux correspondre au sémantisme de l'énoncé, la continuité lui étant peut-être associée d'emblée cependant, notamment en raison de la présence de people qui renvoie à une pluralité.

(276)People had sinned against morality.

(27c)People had sinned against Catholicism, French law, morality.

$\rightarrow$ Les deux énoncés reproduisent parfaitement la situation de (27a).

(27d) He had sinned against Catholicism, French law, morality.

39

$\rightarrow$ On voit ici que le changement de sujet (27a et b) montre que la continuité est en réalité liée à l'alliance du sujet he (qui évoque une détermination précise pour la narration) avec les termes Judaism, American law et morality. Ces trois réalités constituent l'environnement dans lequel évolue le héros depuis le début du roman (il est juif, immoral et émigré aux Etats-Unis). Cette continuité se dégage en outre de l'effet de pluralité consécutive à 
l'énumération de ces trois termes. Il est néanmoins nécessaire de "rechercher », de construire cette addition dans la mesure où elle n'est pas marquée. Dans cet énoncé, il n'existe pas de facteur grammatical à proprement parler en faveur de la continuité.

D’autres énoncés portent clairement leur interprétation d'une continuité sur un segment autre que la forme de past perfect, tel (28) :

(28) Like the Jews, the animals had been dragged here from all parts of the world, condemned to isolation and boredom. (I.B. Singer : 53)

(28a)They had dragged the animals $\emptyset$, like the Jews, from all parts of the world, condemned to isolation and boredom.

$41 \rightarrow$ Le fait d'avoir ôté l'adverbe here permet d'interpréter l'énoncé comme renvoyant à une rupture avec $\mathrm{T}_{1}\left(\mathrm{~T}_{3} \rightarrow \mathrm{T}_{2} \neq \mathrm{T}_{1}\right)$, la continuité avec $\mathrm{T}_{1}$ demeurant toujours fort probable. Le passage de la voix passive à la voix active a pour effet supplémentaire de présenter une continuité non plus essentiellement référentielle [(28)] mais énonciative.

(28b)They had dragged the animals here, like the Jews, from all parts of the world, condemned to isolation and boredom.

$42 \rightarrow$ Le rétablissement de here accroît la probabilité de renvoi à la continuité au détriment de la rupture. Cette dernière n'est cependant pas totalement exclue.

(28c) Like the Jews, they had dragged the animals $\emptyset$ from all parts of Europe.

$43 \rightarrow$ L'élimination de condemned to isolation and boredom appelle catégoriquement une valeur de rupture, d'après les conseils anglophones. Ce serait même cette portion de la proposition qui favoriserait le rapport continu à $\mathrm{T}_{1}$, l'appréciation dont elle fait part ne pouvant être émise qu'en $\mathrm{T}_{1}$.

44 Par conséquent, la continuité associée à (28) provient de la conjugaison de trois facteurs, le passif (explicitation de l'état résultant de la validation de la relation prédicative + effet de la thématisation de la notion verbale ${ }^{11}$ ), l'adverbe here qui « accroche » la référence en $\mathrm{T}_{1}$, ainsi que la combinaison de condemned to isolation and boredom et here précisément, dans laquelle condemned to isolation and boredom apparaît comme une exposition de l'état de choses observé dans la situation délimitée par here.

45 Il existe encore des cas dans lesquels, les énoncés exprimant la continuité, celle-ci apparait davantage comme un moyen qu'un but. Un moyen d'exprimer une relation cause-conséquence (donnée comme une propriété du perfect) :

(29) He realized, as the sound grew loud in his ears, that cicadas were shrilling all about him. He had been too absorbed to hear them. (D. Lessing : 29)

En contexte, la continuité de (29) est référentielle.

(29a) ?He had felt too absorbed to hear them.

$47 \rightarrow$ Cet énoncé est douteux dès l'instant où l'on emploie feel. C'est en fait la juxtaposition de feel et de absorbed qui pose problème dans la mesure où le recours à feel suppose par la suite l'utilisation d'un verbe plus «volontaire». $\mathrm{Si}$, par exemple, on prend worry, la production redevient acceptable :

(29a') He had felt too worried to hear them. $\rightarrow$ On peut tout à fait [feel worried] alors que l'on peut plus difficilement [feel absorbed].

Et dans ce cas, $\mathrm{T} 2 \rightarrow \mathrm{T} 1$ et $\mathrm{T} 3 \rightarrow \mathrm{T} 2 \neq \mathrm{T} 1$ sont tous deux possibles. La continuité référentielle, seule valeur possible de (29), disparait.

(29b) He had gone too far to hear them. 
$50 \rightarrow$ La continuité référentielle devient continuité énonciative, mais pour le reste, elle est toujours en concurrence avec la rupture.

(29c) He had walked too far to hear them.

$51 \rightarrow$ Le changement de verbe conduit à la même conclusion.

(29d) He had seemed too absorbed to hear them.

$52 \rightarrow$ La continuité devient référentielle ; la rupture reste une possibilité. Si nous comparons (29d) avec (29a), nous nous apercevons que (29d) est tout à fait recevable, bien que seem, en tant que semi-copule d'état, s'apparente à feel. Cependant, le message transmis est différent d'un énoncé à l'autre. Avec seem, il est question d'un point de vue extérieur, d'un regard opéré par quelqu'un d'autre, et les conditions qui régissent (29d) ne sont plus similaires à celles de (29a). Ce n'est alors plus un problème que d'utiliser un verbe d'état, car celui-ci n'est en rien co-référentiel, donc incompatible, avec absorbed.

(29e) ?He had been so absorbed that he didn't hear them.

$53 \rightarrow$ En contexte, cet énoncé ressortit à une rupture entre $\mathrm{T}_{2}$ et $\mathrm{T}_{1}$; nous le faisons précéder d'un point d'interrogation car (29f) semble préférable :

(29f) He was so absorbed that he didn't hear them.

$54 \rightarrow$ Mais il n'est plus question de past perfect... En revanche, (29f) permet de percevoir le rôle tenu par so. Bien que conjugué au prétérit, l'énoncé renvoie en quelque sorte à une «linéarité » référentielle que transmet so en tant que lien entre les deux segments de la phrase, he was so absorbed et he didn't hear. Ce rôle tenu par so est plus clair si l'on revient au past perfect :

(29g) He had been so absorbed that he hadn't heard them.

$55 \rightarrow$ Continuité et rupture avec $_{1}$ sont toutes deux présentes. Mais ce à quoi tient précisément la continuité, c'est so, qui conditionne hear (la structure syntaxique est une comparative d'excès construite sur la conjonction de subordination corrélée so... that). Il ne peut, par définition, y avoir de rupture entre le conditionnant et le conditionné dans une relation de conditionnement. Les manipulations avec so, et le passage par (29f) et (29g) mettent en évidence le véritable fonctionnement de (29). Too et so ont une influence semblable sur le sémantisme de continuité de ces énoncés.

56 Partant, le véritable facteur déclencheur de la continuité en (29) est en réalité l'adverbe too qui introduit de même une comparative d'excès et indique une continuité participant d'une relation de cause à effet. La condition pour entendre, c'est effectivement de ne pas être absorbé par autre chose. Il est bien question d'une antériorité de be absorbed par rapport à hear, mais celle-ci est causale et non pas temporelle. Il s'ensuit qu'elle conduit à l'établissement d'une continuité tendant nécessairement vers une conséquence. C'est tout simplement l'équilibre logique de l'énoncé qui est en jeu ici, ce que signale had-en.

57 Il se peut également, et c'est précisément là que réside l'intérêt de l'énoncé, que la continuité soit présente contre toute attente. Le past perfect change alors les données pragmatiques existantes (30):

(30) He paused. 'Tell you what I'll do, Lennie. First chance I get I'll give you a pup.

Maybe you wouldn't kill it. That'd be better than mice. And you could pet it

harder.'Lennie avoided the bait. He had sensed his advantage. (J. Steinbeck 1974 :17)

$58 \rightarrow$ La continuité ressortit ici à autre chose qu'au grammatical. Certes, avec sense, verbe d'état, la continuité est privilégiée. Mais ce n'est pas sur ce constituant qu'elle joue. (30) traduit un revirement de situation. L'énoncé dit qu'en $\mathrm{T}_{1}$, l'on s'attendait à ce que Lennie 
morde à l'hameçon, et il ne l'a pas fait. Ce changement de perspective est d'autant plus significatif que Lennie est le simple d'esprit du roman, auquel on doit dicter le moindre de ses mouvements. Or, contrairement à l'attente des autres personnages, il a fort intelligemment évité de se faire piéger. Le past perfect souligne donc un hiatus d'ordre pragmatique, dans le même temps qu'il semble étayer une relation logique traduite en syntaxe (conséquence: Lennie avoided the bait / had-en de cause: He had sensed his advantage). Cependant, sense ne peut expliquer avoid the bait puisque Lennie n'en a pas les capacités intellectuelles. La continuité tient donc au fait que deux procès qui ne devraient pas être liés sont bel et bien présentés comme étant liés (une cause entrâne une conséquence dans l'extralinguistique - $c f$. 29). Elle provient avant tout de ce lien cognitif (et non uniquement du sémantisme de sense), là où le past perfect rappelle le caractère incongru de la relation prédicative [he - sense the bait]. Ce faisant, il focalise sur un procès dont la notion est différente de celle escomptée et dont la mention renverse les attentes nourries en $\mathrm{T}_{1}$.

59 Voyons encore (31) et (32) :

(31) I don't think I wanted to be a hero, a charioteer of the skies. My father was a hero. I didn't worship my father. But I had wanted to fly. (G. Swift 1988 : 46)

En contexte nous avons une continuité référentielle.

(31a) But I had asked / learnt / come to fly.

$61 \rightarrow$ Le recours à ask, learn ou come rend la continuité principalement énonciative et introduit une rupture référentielle avec $\mathrm{T}_{1}\left(\mathrm{~T}_{3} \rightarrow \mathrm{T}_{2} \neq \mathrm{T}_{1}\right)$.

(31b) But I had needed to fly.

$62 \rightarrow$ La nature statique du verbe réintroduit une continuité référentielle.

(31c) But I had expected to fly.

$63 \rightarrow$ Le passage de want à expect affirme le rôle de but dans l'énoncé. Adversatif, le connecteur signifie que l'on empêche le personnage de voler. Partant, ce que souligne en vérité le past perfect sur want, ce n'est pas l'antériorité mais l'alternative théorique suivante : soit il n'est plus possible de voler, soit le personnage n'a pas volé. Il se trouve que c'est la seconde solution qui est illustrée en contexte : l'énoncé apparaît après un refus émanant de la RAF d'enrôler le héros du roman. Aussi le véritable sémantisme de (31) est-il : « Mais moi, jevoulais voler. »

64 Ce qui apparaît donc, c'est à nouveau une focalisation, sur la différence entre le désir et la réalité de voler, à partir du procès want. La relation prédicative $[I-f l y]$ n'a pas été validée, cependant le désir s'y rattachant subsiste en $\mathrm{T}_{1}$, indépendamment de la réalité. D'où la valeur de continuité. Il est à noter qu'un prétérit sur want n'aurait pas cet effet de dissociation des deux plans du désir et de la réalité.

(32) Then suddenly she smiled tenderly and took me in her arms. [...] We were alive; my father was dead. She had taken Sam into her life. She had known what she was doing; she had made her choice. The fierceness, the frankness of her will to live! (G. Swift $1992: 23$ )

(32a) She had known Ø.

$65 \rightarrow$ Tel quel, l'énoncé peut renvoyer à de la continuité ou à de la rupture.

(32b) She knew what she was doing.

$66 \rightarrow$ La différence entre (32) et (32b) est très intéressante. Avec le prétérit, l'énoncé est dénué de valeur d'intention. Rien ne laisse transparaitre une quelconque préméditation, 
comme tel est le cas dans le texte. On est dans le rapport objectif d'un état de fait en $\mathrm{T}_{1}$ : « elle savait ce qu'elle faisait ».

Le passage du prétérit au past perfect induit une variation sémantique de la notion verbale. Il ne s'agit plus de [savoir] mais d'[être consciente], voire de [faire exprès]. On n'est plus dans le rapport objectif. Le past perfect sur know montre la préméditation: le personnage a réfléchi à ce qu'elle allait faire et l'acte est prépensé. Certes, le personnage est dans la phase résultative de son acte à l'instant où elle l'accomplit, mais là n'est pas l'important. L'important est que cet acte correspond à un choix, difficile à faire qui plus est (choix de se remarier dans le Paris de l'après-guerre avec un homme plus jeune qu'elle peu de temps après le suicide de son mari) et qui par conséquent revêt un caractère subversif en contexte.

68 A nouveau, le message à transmettre est une certaine valeur d'un procès par opposition à sa valeur universelle, ce que rend le past perfect . La focalisation effectuée est contrastive parce qu'elle montre une notion du procès qui s'éloigne de celle qui le définit d'ordinaire.

69 (31) et (32) indiquent que le past perfect permet effectivement d'attirer l'attention sur un point particulier, afin d'indiquer au co-énonciateur sur quoi se concentrer.

70 Chose remarquable, ce trait est commun à la continuité comme à la rupture.

\section{2. la rupture pragmatique( $14 \%$ des énoncés illustrant la rupturesur un total de 69 énoncés)}

71 Pour ce qui est de la rupture, on observe que cette valeur est construite (13 facteurs déclencheurs différents ${ }^{12}$ ) et travaillée. Elle est donc tangible parce que différentielle.

72 Il apparaît en outre que, comme tel est le cas pour la continuité, la valeur de rupture relaie une focalisation sur la notion verbale et ce, à des fins explicatives (non pas de la relation entre les procès mais des présupposés). Pour preuve ces manipulations sur l'énoncé (33) :

(33) He was beautiful, the old man remembered, and he had stayed. (E.Hemingway

:41)

(33a) He stayed, the old man remembered, and he was beautiful.

$73 \rightarrow$ Le prétérit peut très bien rendre la rupture, en collocation avec remember. Le past perfect de (33) est donc le signe d'autre chose.

(336) He had been beautiful and had stayed, the old man remembered.

$74 \rightarrow$ Had-en sur be explicite la rupture reprise plus loin par remember.

(33c) He was beautiful $\varnothing$ and he had stayed.

$75 \rightarrow$ Nous avons là l'expression de la continuité et de la rupture. Aucune n'est plus marquée que l'autre.

(33d) * He ate fish $\varnothing$ and he had stayed.

$76 \rightarrow$ Les deux verbes appartenant à un champ sémantique différent, on les interprète instinctivement comme ne relevant pas d'une même sphère temporelle. (33d) donne l'impression d'être la réunion, sémantiquement non justifiée, de deux mini-énoncés. Notre expérience nous a enseigné que ces deux verbes ne seraient pas a priori réunis, encore moins à l'aide de and relateur.

(33e) He ate fish and he was beautiful.

$77 \rightarrow$ Le recours uniforme au prétérit rend l'énoncé acceptable. 
(33f) *He ate fish and he had been beautiful.

$78 \rightarrow$ L'irrecevabilité de (33f) tient au décalage, inexpliqué, entre deux sphères temporelles, et non pas à la nature des verbes (puisque (33e) est correct).

79 Ces manipulations indiquent que la rupture en (33) est en réalité liée à remember [puisqu'elle réapparaît en (33a)]plus qu'à la forme composée elle-même, qui vise un autre objectif. La différence de temps sur les verbes peut certes s'expliquer par leur aspect interne, mais un autre phénomène intervient : had-en sur stay témoigne davantage d'un dérapage lors de l'élaboration de l'énoncé. Had stayed est en effet construit par rapport à had left qui était ce à quoi l'énonciateur s'attendait en situation et signifie avant tout : « contrairement à mon attente, il - le requin - n'est pas parti. »

80 Le past perfect, en focalisant sur la notion verbale, remet en cause le présupposé que l'énonciateur avait d'emblée associé à l'énoncé, tout comme il permet de révéler la nature de ce présupposé.

81 La focalisation sur le procès peut encore relever d'un conflit, être employé à des fins polémiques :

(34) After Yadwiga's father died, her mother had taken a second husband, who beat his stepchildren (I.B. Singer : 8)

$82 \rightarrow$ Une suite died, took, beat aurait été possible, quoique plus neutre. L'emploi du past perfect permet à nouveau de souligner un hiatus : le point de vue réprobateur de Yadwiga qui, en contexte, reproche effectivement à sa mère son remariage avec un homme violent. Cela est dû à la suite past perfect - prétérit (had taken - beat) laquelle, en présentant les deux procès comme consécutifs, a pour effet de suggérer que la mère de Yadwiga a impatiemment attendu le décès de son premier époux pour prendre le second. Effectivement, deux phases intermédiaires importantes font défaut par rapport à l'ordre logique des choses, ce sont had died et take. Il s'ensuit que la narration procède par ellipse et teinte l'énoncé de rapidité, voire de hâte, augmentant ainsi le ressentiment de Yadwiga envers sa mère (c'est comme si elle sous-entendait : « ces choses-là ne se font pas. »).

\section{Conclusion}

83 Les valeurs du past perfect, longtemps décrites en termes d'antériorité et d'accompli, puis plus récemment de commentaire, d'explication et de bilan, satisfont également à une catégorisation objective selon les concepts de continuité et de rupture dont l'avantage est de rendre compte de la totalité des emplois de la forme composée dans la mesure où ils s'appliquent au référentiel comme à l'énonciatif. Le recours à ces concepts permet de prendre un certain recul par rapport aux présentations aspectuo-temporelles de had-en et met ainsi en évidence une autre valeur de la forme composée, relayée par un phénomène de focalisation sur la notion verbale dénotée par -en qui souligne le fait que la notion verbale retenue est tout autre que celle attribuée d'ordinaire au procès. Had-en est alors un moyen dont dispose le code linguistique pour renvoyer au niveau pragmatique des présupposés ${ }^{13}$.

Cet effet de focalisation à portée pragmatique pourrait être ce qui rapproche véritablement present perfect et past perfect ${ }^{14}$. Par conséquent, le past perfect aurait bien une valeur propre, autre qu'aspectuelle ou temporelle, dans la mesure où il peut tout à fait apparaître dans des contextes qui lui sont a priori étrangers sur le plan de la référence temporelle, comme tel est le cas pour le present perfect ${ }^{15}$. Le past perfect est par conséquent 
apte, dans certains de ses emplois, à refléter une construction toute intellectuelle ${ }^{16}$ de la réalité, légitimée par les expériences de vie partagées par l'énonciateur et le coénonciateur au sein d'un même groupe, à des fins pragmatiques de clarification, plutôt qu'une représentation codifiée de la chronologie extralinguistique.

Il est alors tout à fait remarquable de constater que ce type d'emploi (à portée pragmatique) se retrouve avec le plus-que-parfait en français chez certains enfants, les enfants intellectuellement précoces ${ }^{17}$, et ce très tôt (parfois à partir de 2 ans pour certains). Leur âge est en effet nettement en-deçà de celui auquel les enfants sont capables de conceptualiser la double antériorité liée au plus-que-parfait (même si l'on est en droit de supposer que les sujets précoces acquièrent plus rapidement ou différemment, la notion de temps) ${ }^{18}$, donc l'emploi de la construction ne peut être temporel. La forme composée, ceci vaut également pour le passé composé ${ }^{19}$, ne leur pose cependant aucun problème ${ }^{20}$. Partant, cet emploi focalisant (que l'on retrouve en anglais comme en français) qui sert prioritairement des fins de justification, de renchérissement et parfois de polémique pourrait être une caractéristique de cette forme composée dont l'étude devrait sans doute comporter à l'avenir un triple point de vue: temporel, aspectuel et pragmatique.

\section{BIBLIOGRAPHIE}

BOUSCAREN, J. (1982), «La forme had-en ou past perfect », in Cahiers de Recherche en Grammaire Anglaise, 1, Gap, Ophrys, p. 75-114.

BRACOPS, M. (2006), Introduction à la pragmatique. Les théories fondatrices : actes de langage, pragmatique cognitive, pragmatique intégrée, Coll. Champs linguistiques, de Boeck, Bruxelles.

BRONCKART, J.P. (1976), Les modes d'expression de l'aspect dans le langage de l'enfant, Thèse présentée à la Faculté de Psychologie et des Sciences de l'Education de l'Université de Genève, mention Psychologie génétique, Bruxelles, Dessart et Mardaga.

COTTE, P. (1987), «Réflexions sur l'emploi des temps du passé en français et en anglais à la lumière de deux évolutions récentes du système verbal de l'anglais », Contrastes, 14.15.

FERREIRO d'ARGENTINE, E. (1971), Les relations temporelles dans le langage de l'enfant, Thèse présentée à l'Ecole de Psychologie et des Sciences de l'Education de l'Université de Genève, mention psychologie génétique, Genève, Droz.

GRUBAR, J.C. (2002), « Plaidoyer pour l'intelligence », in Cerveau et développement de l'Enfant Précoce , Congrès de l'AFEP, Paris, p. 40-44.

GUILLEMIN-FLESCHER, J. (2006), « Aspect et détermination : un cas particulier », in Complétude, cognition, construction linguistique, Claude Delmas (éd.), Paris, Presses de la Sorbonne Nouvelle, p. 211-221.

LE GUAY, E. (1995), Recherches sur le Past Perfect en anglais contemporain. Thèse non publiée, Université de Paris IV - Paris Sorbonne, 430 pages. 
PLANCHE, P. (1998), « La construction des notions spatiales chez les enfants intellectuellement précoces, âgés de six à huit ans ", in Enfance, 2, Paris, PUF, p. 159-171.

PLANCHE, P. (1999), « Les stratégies de décentration spatio-cognitives chez les enfants intellectuellement précoces de 6 ans. ", in Bulletin de Psychologie, tome 52 (4), 442, Paris, PUF, p. 473-480.

PLANCHE, P. (2000), « Le fonctionnement et le développement cognitifs de l'enfant intellectuellement précoce ", in L'année psychologique, 100, Paris, PUF, p. 503-525.

ROUSSEL, E. (1999), « She had been healthy and beautiful when he married her ", in Anglophonia/ Sigma $n^{\circ}$ 6, Université de Toulouse-Le Mirail, Presses Universitaires du Mirail, Toulouse, $p$. 235-258.

ROUSSEL, E. (2005a), « De la valeur du commentaire passif anglais », inParcours linguistiques, Domaine anglais, études réunies par Geneviève Girard-Gillet publiées sous la responsabilité de Louis Roux, C.I.E.R.E.C. Travaux 122, Saint-Etienne, Publications de l'Université de Saint-Etienne, p. 271-288.

ROUSSEL, E. (2007b), Forme composée et fonctionnement cognitif, à paraître.

ROUSSEL, E. (2007d), Point de vue neurolinguistique sur des réalisations linguistiques, à paraître in Anglophonia/Sigma $\mathrm{n}^{\circ}$ 22/2007, Université de Toulouse-Le Mirail, Presses Universitaires du Mirail, Toulouse.

\section{ANNEXES}

\section{Source des exemples}

BOUSCAREN, J. (1982), «La forme had-en ou past perfect », inCahiers de Recherche en Grammaire Anglaise, 1, Gap, Ophrys, p. 75-114.

GREENE, G. (1971), The Confidential Agent, Penguin, Londres.

HARDY, T. (1972), « The Distracted Preacher », in The Second Penguin Book of English Short Stories / sous la direction de Christopher Dolley, Londres, Penguin, p. 11-70.

HARTLEY, L.P. (1958), The Go-Between, Londres, Penguin.

HEMINGWAY, E. (1989), The Old Man and the Sea, Londres, Grafton.

KIPLING, R. (1972), "William the Conqueror », inThe Second Penguin Book of English Short Stories / sous la direction de Christopher Dolley, Londres, Penguin, p. 71-104.

LAWRENCE, D.H. (1948), Sons and Lovers, Londres, Penguin.

LESSING, D. (1989), The Grass is Singing, Londres, Paladin.

MURDOCH, I. (1986), The Good Apprentice, Londres, Penguin.

NABOKOV, V. (1980), Lolita, Londres, Penguin.

ORWELL, G. (1989), Coming up for Air, Londres, Penguin.

SINGER, I.B. (1989), Enemies, A Love Story, New York, Signet.

STEINBECK, J. (1974), Of Mice and Men, Londres, Pan. 
SWIFT, G. (1982), Shuttlecock, Londres, Penguin.

SWIFT, G. (1988), Out of this World, Londres, Penguin.

SWIFT, G. (1992), Ever After, Londres, Picador.

WAUGH, E. (1988), A Handful of Dust, Londres, Penguin.

\section{NOTES}

1. Je remercie les deux relecteurs anonymes de la revue pour leurs remarques. Je suis seule responsable des erreurs ou omissions qui pourraient subsister.

2. ".. ce qui laisse envisager que cette forme est utilisée pour autre chose que pour marquer l'antériorité.» (J. Bouscaren 1982 : 77) ou encore: " La forme had-en permet de revenir sur n'importe quel aspect d'un procès passé afin de commenter tel ou tel phénomène. En introduisant cette notion de commentaire, nous pouvons ainsi caractériser globalement la stratégie de l'énonciateur quand il utilise la forme had-en. » (ibid: 91). Ce qu'illustre par exemple l'énoncé suivant : Ehrich stopped dead, his swagger gone. Before his very eyes something magical had happened! He - Ehrich Weiss - had seen it. A huge bouquet of flowers had been created out of nothing by magic!... (The Great Houdini par J. Bouscaren 1982 : 94)

3. E. Le Guay 1995, E. Roussel 1999. Les termes de « continuité » et de « rupture » sont propres au cadre de l'étude présentée ici. La continuité reflète une continuité (référentielle ou énonciative) du procès jusqu'au repère considéré ; la rupture (ou « interruption du procès» - « rupture » évite l'ambiguïté du terme "discontinuité »), reflète une rupture toujours référentielle - ie antériorité temporelle - qui peut être sous-tendue par une continuité énonciative - ie état résultant - entre le procès et le repère considéré. Nous verrons ici que cette rupture peut précisément être autre que temporelle tout en ayant l'apparence d'être avant tout temporelle; c'est la raison pour laquelle nous avons adopté ce terme.

4. Corpus de 15508 occurrences d'anglais contemporain (nouvelles et romans, la presse et les pièces de théâtre illustrant essentiellement le discours rapporté), 4481 sélectionnées pour leur représentativité du comportement de la forme (100 premières pages des supports), avec analyse en termes de repérage puis analyse des facteurs déclencheurs du past perfect; étude onomasiologique et sémasiologique de 1746 occurrences types et examen approfondi du contexte de 69 énoncés en vue de déterminer ce qui peut modifier l'interprétation. C'est lors de cet examen que sont apparues les occurrences focalisantes.

5. Le terme " continu » est délibérément choisi en ce que la linéarité sous-jacente à la relation pourrait être due à une propriété cognitive liée au langage (E. Roussel 2007d à paraitre). D'où également ceux de "continuité » et de "rupture » qui permettent de rassembler les différentes relations objectives relevées en un système binaire, lequel, à l'instar de J. Bouscaren (cf. supra note 2 : «nous pouvons ainsi caractériser globalement la stratégie de l'énonciateur quand il utilise la forme had-en »), permet de préciser la stratégie de l'énonciateur quand il utilise le forme had-en. Voir également infra : «le commentaire relevé dans les années 1980 procéderait de cette rupture ».

6. Ces derniers s'ordonnent de la droite vers la gauche, si l'on s'accorde à représenter ainsi

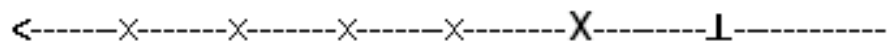

l'antériorité :

$\begin{array}{llllll}T_{5} & T_{4} & T_{3} & T_{2} & T_{1} & T_{0}\end{array}$

7. Contexte de l'énoncé : un agent du gouvernement britannique en charge de récupérer les bébés orphelins et d'organiser leur ravitaillement lors d'une famine à Madras entame une 
conversation avec un ami, conversation qui lui fait oublier ses obligations dont il prend conscience après un moment.

8. Dans ce type d'emploi, l'association du sujet (de l'objet ou d'un déterminant) et du verbe en had-en est susceptible de conduire alors à une recatégorisation aspectuelle du verbe, dont le sémantisme de base se trouve modifié. Ainsi (18) qui dénote une occurrence emblématique comme me l'a suggéré l'un de mes relecteurs. Autre exemple en faveur d'un contraste signifiant sur la notion verbale rendu possible par le past perfect. La recatégorisation observée en (18) ne se produirait en effet pas avec un prétérit : Marie-Antoinette said of the poor, 'Can't they eat cake?'.

9. Expression adverbiale, logique contexto-grammaticale, sémantisme interne des verbes, réitération d'occurrences d'un (ou de plusieurs) procès, ou d'une (ou de plusieurs) situation(s) référentielle(s), focalisation sur la notion du procès conjugué au past perfect, valeur résultante consécutive à l'accomplissement du procès.

10. E. Le Guay 1995, p. 169. Réflexion faite par les conseils anglophones sollicités pour l'étude. Les manipulations qui suivent ont toutes fait l'objet d'un minutieux travail de vérification en leur compagnie.

11. E. Roussel 2005a.

12. Qui se déclinent selon les groupes suivants : expression adverbiale, proposition subordonnée en when, proposition subordonnée relative, détermination marquée, choix des sujets, jeu sur le sémantisme interne des verbes, effet de la pragmatique (contextuelle ou extralinguistique).

13. La présupposition dans le past perfect est, différemment, prise en compte par J. GuilleminFlescher 2006.

14. Cette focalisation sur la notion verbale propre au present perfect a été montrée par P. Cotte 1987.

15. Les exemples de present perfect en collocation avec des expressions d'ordinaire liées au renvoi au révolu sont de plus en plus fréquemment rencontrées.

16. -Ed sur have ne serait alors pas temporel dans ces constructions (E. Roussel 1999).

17. Sujets dont l'éloquence (entre autres) est liée à des dispositions cognitives qui leur sont spécifiques, notamment un recours plus important à l'hémisphère droit (siège des émotions et de la mémoire des expériences individuelles) que les sujets non précoces. L'observation de leurs compétences peut sans doute permettre au linguiste de mieux comprendre certains fonctionnements du langage ; ce point de convergence entre l'emploi du past perfect décrit ici et l'emploi du plus-que-parfait que peuvent faire ces enfants est l'objet d'un travail à paraitre (E. Roussel 2007d).

18. Sur le plan cognitif, l'enfant maîtrise la notion de temps vers l'âge de 7 ans (niveau des opérations concrètes de J. Piaget, qui permet la conceptualisation de la réversibilité nécessaire à la prise en compte de l'accomplissement total d'une action et à sa mise en relation avec le moment de l'énonciateur). Avant cet âge (période pré-opératoire : 2 ans - $6 ; 7$ ans) la réversibilité n'est pas en place. La double antériorité quant à elle peut être problématique jusque vers 10 ans. Ceci vaut pour les enfants non précoces. Cependant, des études sur l'acquisition des notions spatiales chez les précoces (P. Planche 1998, 1999, 2000) ont montré que même si le mode de développement cognitif des précoces est "marqué d'aspects irréguliers et dyssynchroniques " (P. Planche 2000 : 524), le moment de développement de ces notions est approximativement semblable aux non-précoces. Seule la vitesse d'acquisition des connaissances (plus rapide chez les précoces) diffère une fois le processus amorcé. Ces enfants se distinguent par le fait qu'«ils mettent en œuvre des attitudes et des caractéristiques fonctionnelles qui attestent d'un contrôle de l'activité cognitive plus soutenu que chez d'autres sujets [...] La qualité de fonctionnement qui caractérise les enfants précoces influence leur rythme de développement. » (P. Planche 2000:521 et 522). Or, nous savons que le développement de la notion de temps est contemporain de celle de l'espace. Donc, ces enfants n'acquièrent pas plus tôt les notions temporelles. La question est alors de savoir si leurs spécificités cognitives (qui s'illustrent entre autres par cet emploi pragmatique 
de la forme composée) sont ou resteront typiques de leur fonctionnement, ou si elles peuvent, d'une manière ou d'une autre, influer sur notre connaissance de la gestion du temps (aussi bien sur le plan cognitif que sur celui de sa codification) par les non-précoces. Pour preuve la similitude observée avec les emplois de past perfect étudiés ici et attribués a priori à des sujets non-précoces (c'est moi qui souligne).

19. E. Roussel 2007b, à paraître.

20. E. Ferreiro (1971) puis J.P. Bronckart (1976) ont déjà montré qu'avant 7 ans les enfants assignent aux formes temporelles du français une valeur essentiellement aspectuelle et qu'ils peuvent uniquement conceptualiser la simultanéité d'actions.

\section{RÉSUMÉS}

L'article traite de certaines occurrences de past perfect dont la valeur est en réalité différente de celle qui semble être la leur a priori. Le past perfect y présente la particularité de permettre à l'énonciateur de distinguer une notion verbale comme étant surprenante en contexte ou non conforme aux attentes. D'où l'intérêt de produire l'énoncé dont la portée est alors essentiellement pragmatique. Dans de telles occurrences, les valeurs d'antériorité, de résultat ou de bilan d'ordinaire associées au past perfect passent au second plan.

This paper puts forward a new analysis of the past perfect structure. The careful study of various utterances reveals that it can have a clearly pragmatic scope. The compound form appears as a means of underlining a semantic shift of the verbal notion. What matters then is to show that this verbal notion does not tally with the speaker's or the addressee's expectations. The pragmatic background governing the construction of the utterance has become inadequate in context. The past perfect can operate as a way for the speaker to use the linguistic code in order to refer to the pragmatic level of presupposed items.

\section{INDEX}

Mots-clés : continuité, rupture, pragmatique, focalisation, notion verbale, présupposés

\section{AUTEUR}

\section{EMMANUELLE ROUSSEL}

Université de Caen - CRISCO (FRE 2805) 